\title{
Wall pressure signatures of turbulent flow over longitudinal
}

\author{
Hayder A. Abdulbari ${ }^{1,2}$, Hassan D. Mahammed ${ }^{1}$, Zulkefli B. Yaacob ${ }^{1}$ and Wafaa K. Mahmood ${ }^{2}$ \\ ${ }^{1}$ Faculty of Chemical and Natural Resources Engineering, Universiti Malaysia Pahang, Gambang 26300, Kuantan, Pahang, Malaysia \\ ${ }^{2}$ Center of Excellence for Advanced Research in Fluid Flow, Universiti Malaysia Pahang, Gambang 26300, Kuantan, Pahang, Malaysia
}

\begin{abstract}
Five triangular riblets longitudinal in the streamwise direction have been studied experimentally. The riblets have pick to pick spaced (s) equal to $1000 \mu \mathrm{m}$ and with groove height to space ratio (h/s) $0.4,0.6,0.8$ and 1 . The tests were conducted in a full turbulence water channel on a flat plate for Reynolds numbers 13000 to 53000 based on channel hydraulic diameter. Pressure drop was measured using pressure transmitter gauge with pressure tap points of $12.7 \mathrm{~mm}$ in diameter were provided at the bottom of the channel. The main purpose of the present study is to investigate the response of turbulent flow to longitudinal grooves of triangular shaped riblets and compare the effect of the turbulence structure over smoothed and grooved surfaces with pressure drop measurements. 10.20 was the maximum drag reduction appear at $\mathrm{h} / \mathrm{s}$ equal to (1).
\end{abstract}

\section{Introduction}

The effects of rib surfaces on the turbulent flow in rectangular channels and circular tubes have been extensively studied in literature [1-10]. Several challenges face surface textures and disabling this technology to be translated into industrial applications.

Choi [11] studied the wall pressure fluctuations in a wind tunnel to modified turbulent boundary layer with riblets. The tunnel had a test section of $(4.8 \mathrm{~m} \times 2.4 \mathrm{~m} \mathrm{x} 15 \mathrm{~m})$ and $0.5 \%$ flow speed controlled by a feedback control system. The results of this study were drawn comparing with smooth surface. The results revealed that surface modifications reduced RMS intensity up to $4 \%$. The reduction appeared as a result of decrease in turbulence intensity within a small volume near a riblet surface.

Reidy [12] and Reidy and Anderson[13], conducted experimental studies in $3 \mathrm{M}$ riblets in a high speed water tunnel over a flat plate in turbulent flow. The second experiment has been in 6-inch diameter pipe line system. $3 \mathrm{M}$ vinyl riblets have been used in both systems with height and spacing of $\mathrm{V}$-groove $\mathrm{h}=\mathrm{s}=76.2 \mu \mathrm{m}$. the results shows a maximum drag reduction $8.1 \%$ Nonetheless, several challenges face surface textures and disabling this technology to be translated into industrial applications. Where there are no certain design (shape and dimensions) of riblets that can be used for all type of applications, due to turbulent regime where the turbulence coherent structure are completely random, as the viscous sublayer and the streamwise vortices are different from fluid to another due to properties of the fluid and the application circumstances . In the present work, triangular riblets will be designed, fabricated and tested in a closed loop liquid circulation system. the flow rate will be the controlled variable and the pressure drop across the rib surface will be measured to evaluate the drag reduction performance.

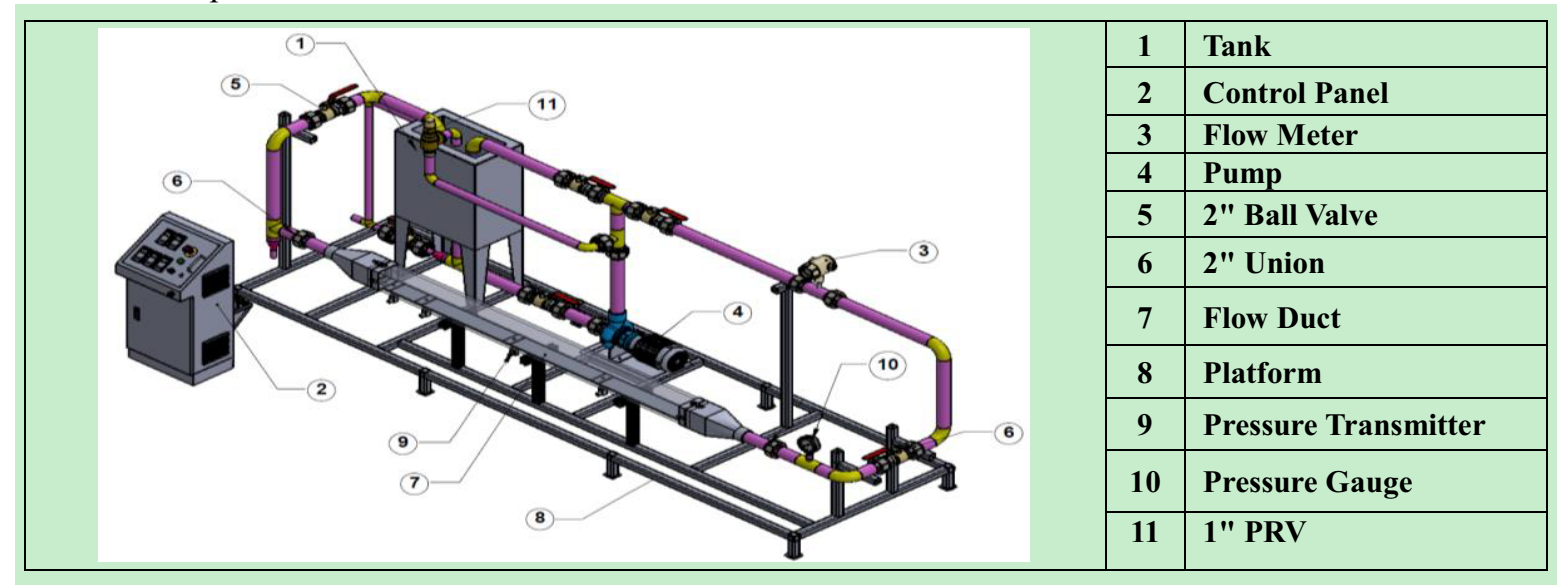

Figure 1. Shows the experimental rig. 


\section{Experimental set-up and measuring techniques}

\subsection{Experimental Apparatus and Riblets Fabrication}

The main purpose of the present work is to determine the effect of longitudinal triangular riblets on the development of the pressure characteristics over the selected models. A closed loop system was used in the present work to test the drag reduction efficiency of the chosen riblet dimensions as shown in Figure 1. The system consists mainly of one tank rectangular in shape and had a length of $500 \mathrm{~mm}$ width of $300 \mathrm{~mm}$ depth of $520 \mathrm{~mm}$ and capacity of 70 litters; pump with capability to drive the primary fluid at a rate of $36 \rrbracket \mathrm{m} \llbracket \wedge 3 \mathrm{hr}$, PVC pipes with 2 inches in size, flow meter, pressure transducers and square channel. Tap water was used and repeatedly changed after each series of tests. Water is then pumped from the centrifugal pump, from the supply tank, into the PVC pipes, then through the transparent channel and into the supply tank again.

The square channel of the experimental rig has been designed and fabricated in such a manner that there are four transparent polycarbonate plates with dimensions $(2 \times 0.12 \times 0.01) \mathrm{m}$, which have been used to fabricate the channel. Acrylic welding was used in fabrication of all the sections of the channel. Four pressure tabs were placed at the bottom surface of the channel that was used to measure the pressure drop. These tabs comprised the test sections, where $0.16 \mathrm{~m}$ was placed after two sections of $0.55 \mathrm{~m}$ to ensure a full turbulence flow. The system also featured six ball valves that were strategically located at the points of entry and exit of the water and were primarily present to regulate the flow of water. The pressure transmitters were connected to the first test section with a length of $0.16 \mathrm{~m}$, and the pressure drop was taken for the section over the smooth plate and rib surfaces. The range of Reynolds numbers was from 13000 to 53000 based on channel hydraulic diameter. Fluctuating pressure measurements in the channel wall were taken with great care to eliminate all vibration in the experimental equipment.

Aluminum sheets with dimensions $(9.53 \times 90 \times$ $3000) \mathrm{mm}$ have been used for manufacturing the riblet surface, the plate first have been cut in to the size of the test section $(8 \times 80 \times 160) \mathrm{mm}$ with $\mathrm{CNC}$ machine. After prepared the plates, WEDM machine was used to fabricate the triangular groove. A Brass Wire with a diameter of $150 \mu \mathrm{m}$ was used as an electrode to erode a wokpiece. The four triangular riblets were fabricated longitudinal to the direction of the streamwise with the characteristic dimensions listed Figure 2 that illustrates grooves that are defined by peak height (h) and peak-topeak spacing (s) with selected dimensions. The groove peak-to-peak spacing was fixed for all fabricated riblets to $1000 \mu \mathrm{m}$ but with height to space ratio $0.4,0.6,0.8$ and 1. The main purpose of the present study is to investigate the response of turbulent flow to longitudinal triangular grooves of with different rib height and fixed rib spaced and compares the effect of the turbulence structure over smoothed and grooved surfaces with pressure drop measurements.

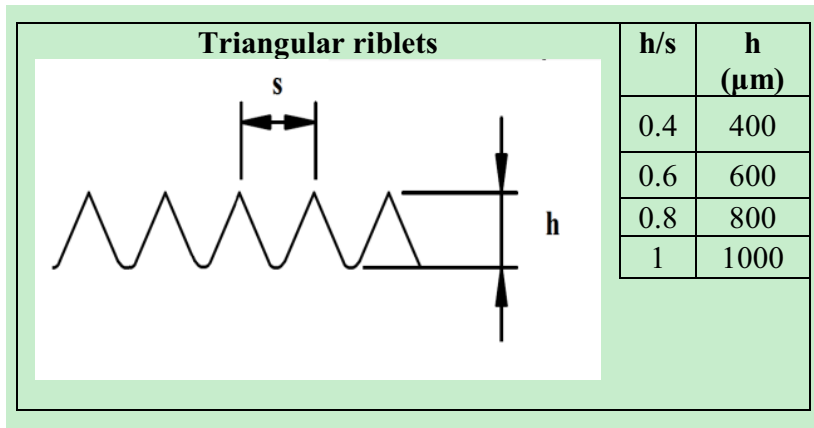

Figure 2. Dimensions of fabricated triangular riblets.

\subsection{Measurements}

The The skin friction coefficients (Cf) and Reynolds numbers $(\mathrm{Re})$ were calculated from the wall shear stress and flow rate measurements by using the following equations:

$$
C f=\frac{2 \tau_{w}}{\rho u^{2}} \text { and } R e=\frac{u D_{h}}{v}
$$

where $(u)$ is mean velocity $(\mathrm{m} / \mathrm{sec}),(v)$ is the kinematic viscosity of water $\left(\mathrm{m}^{2} / \mathrm{sec}\right),(\rho)$ is the density of water $\left(\mathrm{Kg} / \mathrm{m}^{3}\right)$, and $\left(\tau_{w}\right)$ is the wall shear stress in a fully developed pipe flow, as defined by Perry et al. [33]. This equation relates the wall shear stress to the pressure drop during the turbulent flow inside the pipelines.

$$
\tau_{w}=\frac{\Delta p D_{h}}{4 L}
$$

The height and spacing of the grooves in wall units are calculated by using Walsh [34] identification in terms of wall units as non-dimensional height $\left(\mathrm{h}^{+}\right)$and spacing $\left(\mathrm{s}^{+}\right)$, as;

$$
h^{+}=\frac{h u}{v} \sqrt{\frac{C_{f}}{2}} \text { and } s^{+}=\frac{s u}{v} \sqrt{\frac{C_{f}}{2}},
$$

where $\left(C_{f}\right)$ is local skin friction coefficient, $(s)$ is peak to peak spacing of riblets and $(h)$ is the valley to peak height of riblets.

The percentage drag reduction (DR \%) is defined as:

$$
D R \%=\left(\frac{\Delta P_{\text {Smooth }}-\Delta P_{\text {riblet }}}{\Delta P_{\text {Smooth }}}\right) \times 100
$$

The diameter used in the calculation of the Reynolds number and friction coefficient is defined as the hydraulic diameter $D_{h}=4 \frac{A}{p}$, where $(A)$ is the area section of the duct and $(p)$ is the wetted perimeter of the duct.

\section{Results and Discussion}

As per the theory, the effect of longitudinal grooved surfaces on wall-pressure fluctuations can be estimated by calculating the effect first on smooth flat plate and then on ribbed plate. In order to get a well-defined result, 
the Reynolds number and surface roughness of both the plates should be the same.

The first thing that needs to be addressed before measurement is the time series of pressure. We can analyze the time domain by plotting a graph of the sequence of data points of signal obtained. This will give an accurate description of both the time scale, rate and complexity of the flow. The pressure signal, on the other hand, depends on the random fluctuations which are roughly near the pressure mean value.

The turbulent flow relies on fluctuations in three factors, namely, pressure, acceleration and shear stress. All three are directly associated with position and time. These fluctuations directly affect the velocity and pressure terms in the momentum and energy equations which will show varied differences. Figures 3 show the pressure drop fluctuations over the smooth and riblet plates at Reynolds number $5.3 \times 10^{4}$.

There is a mechanism that controls the drag reduction and behavior of the grooved surfaces which needs to be

investigated in the experiment. For that, the pressure drop readings of each type of surface and the flow rate are measured at the same time at $60 \mathrm{~s}$. As a result, there arise two distinct groups of fluctuations that contribute to the wall pressure as per the suggestion provided by the collective body of results.

There are two groups of fluctuations that make up the wall pressure. The first one is made up of large-scale disturbances that are of low-frequency. Such disturbances come from the surrounding portions of the boundary layer and goes up to within the unsteady potential flow.
The large-scale disturbances remain consistently on course with the character of the interface of the potential flow which is outside the boundary layer.

The second group of pressure fluctuation, on the other hand, is made up of small-scale disturbances that are of high-frequency and are considered to have a relation with the burst-sweep cycle of events. Such disturbances are also noted to follow the wall pressure fluctuations of large-amplitude. The large-amplitude wall pressure peaks, according to the results of the conditional sampling techniques on signals of pressure and velocity that are simultaneously acquired, are realized to have relation to the shear layer structures found in the buffer region, and have an association with the bursting process in flat-plate turbulent boundary layers, turbulent flows of pipes as well as direct numerical simulation turbulent channel flows.

The pressure drop performed on the surface showed readings that are stable while having a turbulence frequency lower than other surfaces. It was seen that the pressure drop point is below the smooth surface pressure drop but with irregular readings. Now the structured surfaces exhibit pressure readings which are higher in amplitude and smooth surface readings which are lower in amplitude. It is seen that the structured surfaces have a lower pressure drop on average but in some points, it may be higher. This is due to the fact that the low pressure drop arises when the pressure drops stabilizes for a certain time and then returns to the smooth surface readings. The same pattern repeats several times which explains the low pressure drops on average.

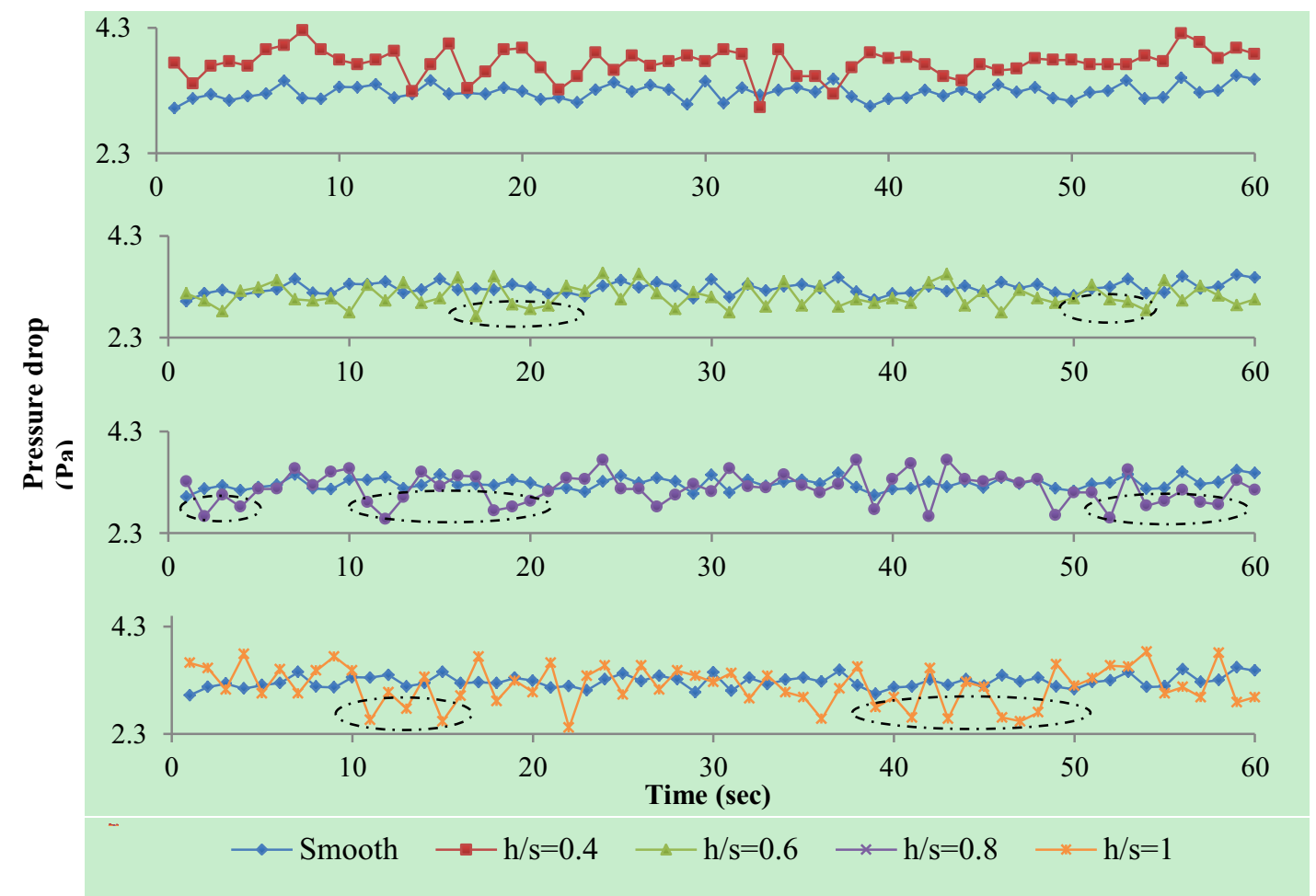

Figure 3. Pressure fluctuation time series at Reynolds number $5.3 \times 10^{4}$ for smooth and triangular riblet.

The profiles of the pressure fluctuation time series under different conditions were illustrated as in Figures 4.
The turbulent characteristics in the square channel were fully embodied. Generally the pressure fluctuation profile 
with different Reynolds numbers at smooth and triangular riblet, the fluctuation of pressure time series obviously increased as the Reynolds number of the inlet flow increased over riblets compared to smooth surface. But the amplitudes average values of riblet is less than amplitudes average values of smooth surface.
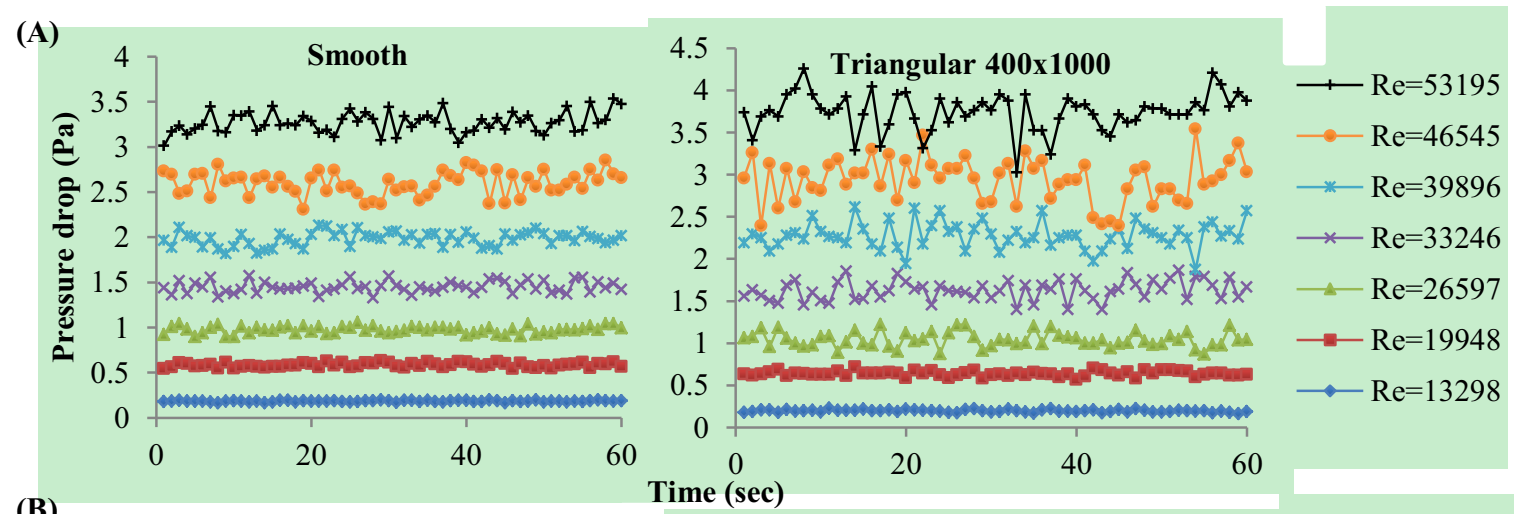

(B)
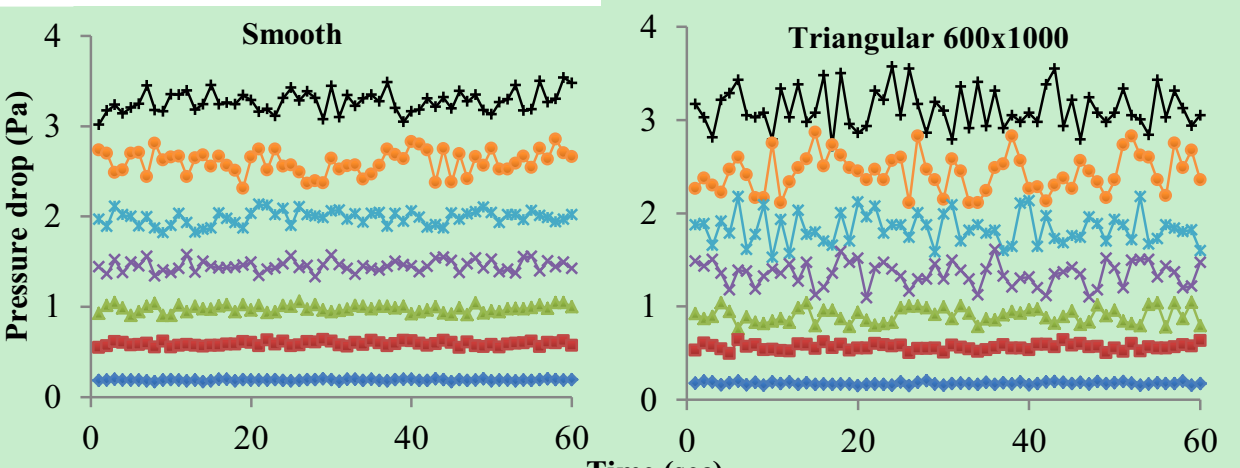

(D)

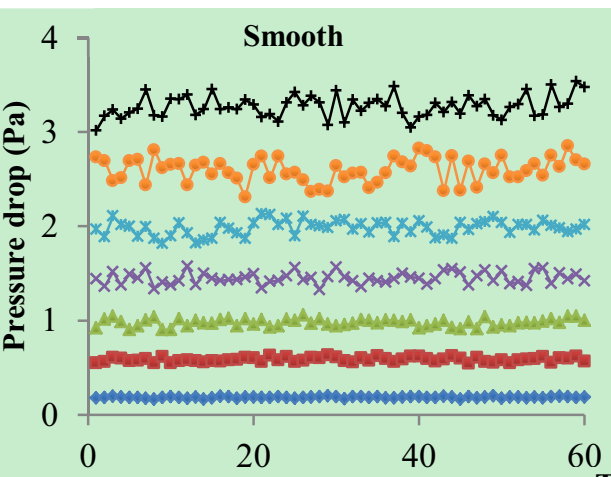

Time (sec)
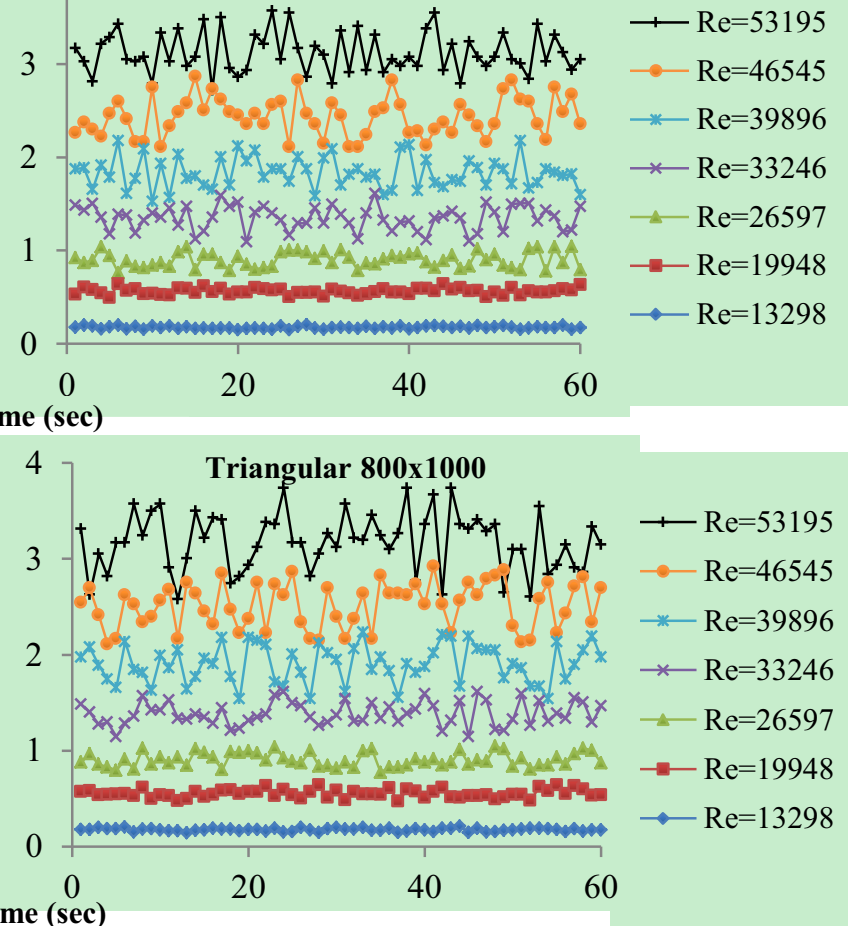

(E)
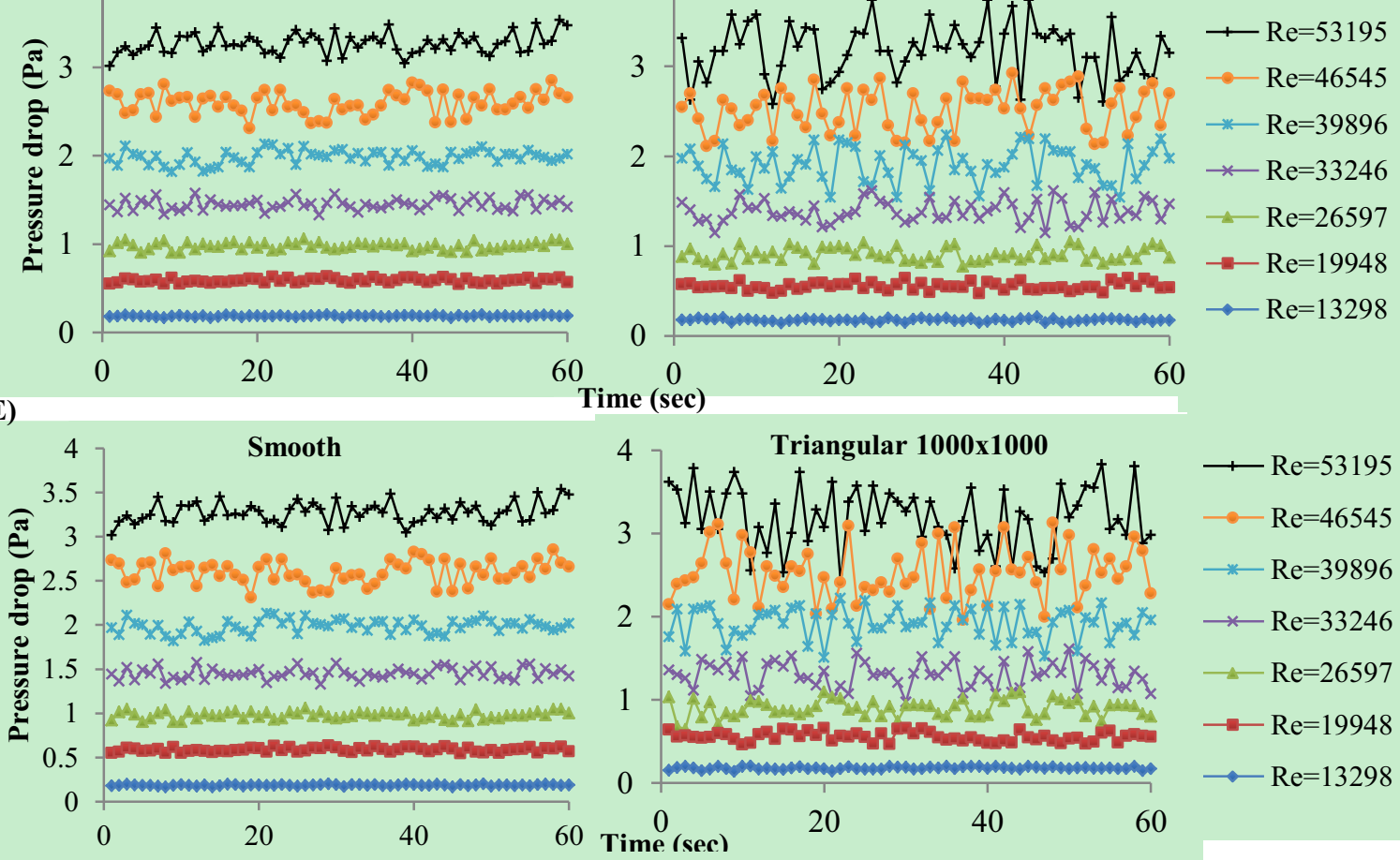
60

Figure 4. The profiles of pressure fluctuation time series under different Reynolds number for smooth and triangular riblet with selected dimensions (A) triangular riblet $400 \times 1000 \mu \mathrm{m}$, (B) triangular riblet $600 \times 1000 \mu \mathrm{m}$, (C) triangular riblet $800 \times 1000 \mu \mathrm{m}$, (D) triangular riblet $1000 \times 1000 \mu \mathrm{m}$.

These experiments deal with the Reynolds numbers between 13000 and 5000. Figure 5 discuss the pressure drop measured across the smooth plate and all triangular riblet surfaces. The initiation happens in between the region of $1.3 \times 10^{4}$ and $1.9 \times 10^{4}$. And as the Reynolds number increases, the pressure drops increase. Afterwards, in the region of $1.9 \times 10^{4}$ to $5.3 \times 10^{4}$, the zone is represented by the pressure drop which continues 
to rise on two characterized zones can be spotted out easily. This are the joining zone where the pressure drop of the triangular grooved surfaces and that of the smooth surface are near a Reynolds number going from $1.3 \times$ $10^{4}$ and $2.6 \times 10^{4}$ are conveniently used to delineate a relentless increment in the rate of pressure drop as the Reynolds number expansions.

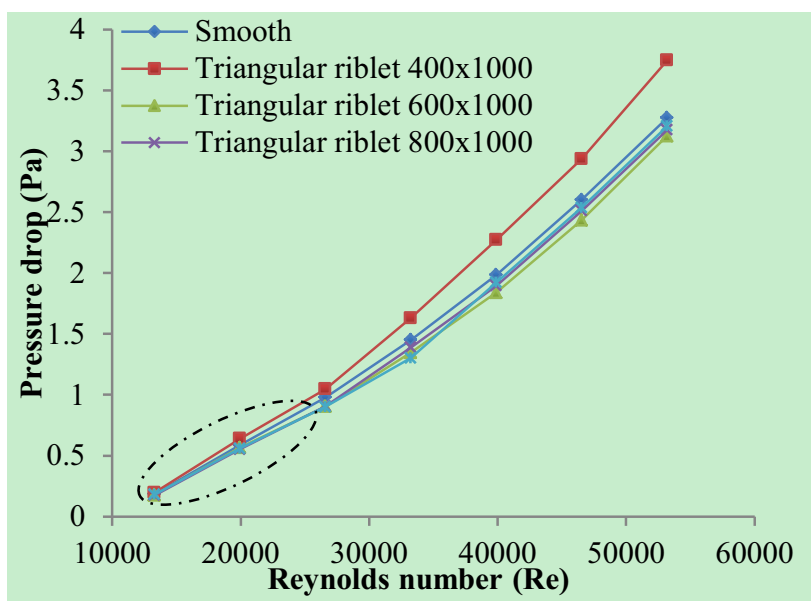

Figure 5. Pressure drop comparison over smooth and triangular groove.

The other defined zone is the pressure drop of the tried plates that has a method for ascending on a consistent premise and begins to separate to the Reynolds number running from $2.6 \times 10^{4}$ to $5.3 \times 10^{4}$. On the hand, it must be noted of that the trends of the three triangular riblet dimensions with height 600, 800 and $1000 \mu \mathrm{m}$ are below the pressure drop of smooth plate which show a capability to reduce the drag force but with triangular riblet $400 \times 1000$ show increasing in pressure drop compare to smooth surface. Figures 6 demonstrate the effect of non-dimensional space $\left(s^{+}\right)$and Reynolds number on the percentage of drag reduction with different triangular riblet heights. the data obtained for Reynolds number range of $\mathrm{Reb} \approx 13000-53000$. The riblet spaces in nondimensional wall units were then estimated from equation (3) and the percentage of drag reduction was estimated from equation (4). The present measurements of riblet drag reduction shows that the percentage drag reduction increases by increasing the $s^{+}$and Reynolds number for most of the tested riblets until a certain value where the percentage drag reduction start to descend. Which means increasing the turbulence spectrum and reaching a maximum point or maximum performance "which might be considered as optimum performance for this point only". After that and by a further increase in the value of the $s^{+}$and Reynolds number start to decline in most cases due to the strong action of these turbulent structures over riblets, where riblets no longer could modify these structures, which is dependent on the properties of the particular boundary layer. However, the best performance for riblets was in range of $S^{+}$equal to 15-20 wall unit and for Reynolds number range equal to 30000 to 40000 , high drag reduction up to $10.20 \%$ was obtained based on repeatable pressure drop measurements.

\section{Conclusions}

Experimentation was carried on triangular riblets with four different sizes of grooves to determine which one shows the maximum drag reduction in a particular water square channel at different Reynolds numbers. The peak to peak spaced dimension was the same for all four tasted riblets $1000 \mu \mathrm{m}$ with height to space ratio was chosen to be 0.4-1. After the thorough experimentation, we can conclude that the triangular riblets show an increase in pressure drop as the Reynolds number increases. The triangular riblet with $1000 \times 1000 \mu \mathrm{m}$ reach maximum drag reduction 10.2 at Reynolds number $3.3 \times 10^{4}$ and then start to decline at Reynolds number $4.2 \times 10^{4}$.

\section{References}

1. H. A. Abdulbari, H. D. Mahammed, and Z. B. Y. Hassan, ChemBioEng Reviews 2, 185 (2015).

2. B. Dean and B. Bhushan, Philosophical Transactions of the Royal Society of London A: Mathematical, Physical and Engineering Sciences 368, 4775 (2010).

3. F. E. Fish and G. V. Lauder, Annual Review of Fluid Mechanics 38, 193 (2006).

4. M. Gad-el-Hak, Applied Mechanics Reviews 49, 365 (1996).

5. A. M. Savill, in Structure of Turbulence and Drag Reduction (Springer Berlin Heidelberg, 1990), pp. 429.

6. H. A. Abdulbari, R. M. Yunus, N. H. Abdurahman, and A. Charles, Journal of Industrial and Engineering Chemistry 19, 27 (2013).

7. M. J. Walsh, in Viscous drag reduction in boundary layers, edited by D. Bushnell, and J. Hefner (American Institute of Aeronautics and Astronautics, Washington, DC, 1990), pp. 203.

8. A. M. Savill, Flow visualization IV 1, 303 (1987).

9. S. P. Wilkinson, J. B. Anders, B. S. Lazos, and D. M. Bushnell, International Journal of Heat and Fluid Flow 9, 266 (1988).

10. P. Vukoslavcevic, J. M. Wallace, and J. L. Balint, AIAA Journal 30, 1119 (1992).

11. K.-S. Choi, in Turbulence Management and Relaminarisation, edited by H. W. Liepmann, and R. Narasimha (Springer Berlin Heidelberg, 1988), pp. 149.

12. L. W. Reidy, Flat Plate Reduction in a Water Tunnel Using Riblets, 1987.

13. L. Reidy and G. Anderson, in 26th Aerospace Sciences Meeting (American Institute of Aeronautics and Astronautics, 1988). 